\title{
Phase I study of docetaxel, cisplatin and concurrent radiotherapy for locally advanced gastric adenocarcinoma
}

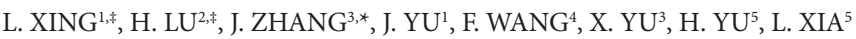 \\ ${ }^{1}$ Department of Radiation Oncology and Shandong Province Key Laboratory of Radiation Oncology, Shandong Cancer Hospital, Shandong \\ Academy of Medical Sciences; ${ }^{2}$ Department of Radiation Oncology, Tianjin Medical University Cancer Institute and Hospital; ${ }^{3}$ Department of \\ Radiation Oncology, Shandong University Affiliated Qianfoshan Hospital; ${ }^{4}$ School of Medicine, Shandong University; ${ }^{5}$ Department of Surgery, \\ Shandong University Affiliated Qianfoshan Hospital
}

*Correspondence: zhangjd165@sina.com

${ }^{\ddagger}$ Contributed equally to this work.

\section{Received October 20, 2011 / Accepted January 15, 2012}

\begin{abstract}
This phase I study is designed to determine the maximal tolerated dose and the dose-limiting toxicity of docetaxel with cisplatin and concurrent radiotherapy in patients with unresectable locally advanced gastric adenocarcinoma.

Docetaxel was given once a week with the dosage escalated from $5 \mathrm{mg} / \mathrm{m}^{2}$ to $15 \mathrm{mg} / \mathrm{m}^{2}$ in increments of $2.5 \mathrm{mg} / \mathrm{m}^{2}$. Cisplatin were administered at $20 \mathrm{mg} / \mathrm{m}^{2}$ once a week. Radiotherapy was delivered to $50.4 \mathrm{~Gy}$ at $1.8 \mathrm{~Gy} /$ day. At least three patients were enrolled at each level. The maximal tolerated dose (MTD) and dose-limiting toxicity (DLTs) was determined. The DLTs were defined as grade 3 or 4 hematologic and nonhematologic toxicity.

Twenty-one patients with locally advanced gastric adenocarcinoma were enrolled. Grade 1-2 neutropenia and nausea/ vomiting were the most common side effects. The first DLT (grade- 3 neutropenia) was observed in one of three patients at $12.5 \mathrm{mg} / \mathrm{m}^{2}$ docetaxel. Three more patients were enrolled, but DLT was not observed and 6 patients were enrolled into $15 \mathrm{mg} / \mathrm{m}^{2}$ group, DLT occurred in 3 patients (1 Grade 3 neutropenia, 1 Grade 4 neutropenia and 1 Grade 3 nausea/vomiting). Overall tumor response rate was $66.7 \%$ with $28.6 \%$ complete and $38.1 \%$ partial response.

In conclusion, the MTD of docetaxel was $15 \mathrm{mg} / \mathrm{m}^{2}$, and the recommended dose of docetaxel for Phase II study was $12.5 \mathrm{mg} / \mathrm{m}^{2}$ weekly. The docetaxel and cisplatin with concurrent radiotherapy were tolerable and feasible in treating locally advanced gastric adenocarcinoma.
\end{abstract}

Key words: gastric adenocarcinoma, phase I trail, docetaxel, cisplatin, radiotherapy

Gastric cancer is one of the most common malignant tumors world widely [1]. Many advances have been made in diagnosis and treatment of gastric cancer in the last decades but the prognosis remains disappointing, especially for disease in advanced stage. Surgical resection offers the only potential for cure in early stage and operable locally advanced gastric cancer. However, approximately $20 \sim 30 \%$ of patients are not resectable owing to locally advanced and distant metastatic disease at diagnosis [2]. If untreated or received supportive cares only, the median overall survival time of these patients is not longer than six months [3].

Patients with unresectable locally advanced gastric cancer are candidates for concurrent chemoradiotherapy, which has shown to be advantageous over radiotherapy alone with improvements of quality of life and survival [4]. Several new active agents and regimens have been studied for gastric carcinoma to improve the responses and survival [5]. Among these regimens, docetaxel has been investigated and demonstrated promising both as monotherapy and in combination with other agents, including cisplatin, 5-fluorouracil or capecitabine, etc [6-8]. Docetaxel and cisplatin (DC regimen, docetaxel $85 \mathrm{mg} /$ $\mathrm{m}^{2} \mathrm{~d} 1$ and cisplatin $75 \mathrm{mg} / \mathrm{m}^{2} \mathrm{~d} 1, \mathrm{q} 3 \mathrm{w}$ ) have been reported to be active in advanced gastric cancer with $56 \%$ response rate in a multicentric trial [9]. Modified DC regimen (docetaxel $15-30 \mathrm{mg} / \mathrm{m}^{2}$ and cisplatin $15-30 \mathrm{mg} / \mathrm{m}^{2}$ per week) combined with concurrent radiotherapy (50Gy) had also been shown feasible for locally advance esophageal cancer in a phase I trail published recently [10]. However, its recommended dose of docetaxel (30 mg/m² per week) is much higher than $7.5 \mathrm{mg} / \mathrm{m}^{2}$ reported in another phase I trail using docetaxel and 5-fluor- 
ouracil with concurrent radiotherapy for esophageal cancer [11]. The feasibility of modified DC regimen combined with radiotherapy for unresectable gastric adenocarcinoma should be explored and the optimal dose of the docetaxel in this setting must be determined.

We conducted the phase I trial with escalating weekly doses of docetaxel which were combined with fixed dose of weekly cisplatin and concomitant radiotherapy for locally advanced gastric adenocarcinoma. The maximal tolerated dose (MTD) of docetaxel defined by this trail is going to be used in further phase II trial.

\section{Patients and Methods}

Patient Eligibility. Eligibility criteria include: (1) histologically confirmed adenocarcinoma of the stomach; (2) unsuitable for surgical resection (due to advanced $\mathrm{T}$ or $\mathrm{N}$ stage, or patient medically unsuitable for surgery) based on multidisciplinary opinion; (3) age 18 70 years; (4) Eastern Cooperative Oncology Group (ECOG) performance status (PS) of $0 \sim 1$; (5) life expectancy of $>3$ months; (6) adequate organ function--bone marrow: $\mathrm{WBC} \geq 4.0 \times 10^{9} / \mathrm{L}, \mathrm{HGB} \geq 100 \mathrm{~g} / \mathrm{L}, \mathrm{PLT} \geq 100 \times 10^{9} / \mathrm{L}$; renal: serum creatinine $<15 \mathrm{mg} / \mathrm{L}$; liver: serum bilirubin level $<15 \mathrm{mg} / \mathrm{L}$, serum AST and ALT within 2.5 times the upper limit of normal; (7) written informed consent. Patients were excluded at following criteria: (1) pregnant or lactating women; (2) history of chemotherapy or radiotherapy; (3) synchronous double cancer; (4) complete gastrointestinal obstruction; (5) active bleeding; (6) severe concomitant illnesses or medical conditions. Institutional ethics committee approval was obtained from each participating center.

Chemoradiotherapy plan. The treatment schedule is outlined in Figure 1. Chemotherapy started on day 1 of radiotherapy. The cisplatin at $20 \mathrm{mg} / \mathrm{m}^{2}$ was administered as 60-90 minutes infusion weekly. Docetaxel was infused over $1 \mathrm{~h}$ once a week with premedicated dexamethasone (10 mg intravenously 30 min prior to docetaxel infusion). Radiotherapy was delivered using the three-five fields three-dimensional conformal radiotherapy (3D-CRT) technique. The gross tumor volume (GTV) included the primary tumor and involved lymph nodes. Total dose of 50.4 Gy was given in 28 fractions of $1.8 \mathrm{~Gy}$ (five fractions per week) with dose prescribed to the isocenter of $95 \%$ of the planning target volume.

Dose levels and treatment modification. The dose of docetaxel started at $5 \mathrm{mg} / \mathrm{m}^{2}$ (level 1), and escalated to $15 \mathrm{mg} / \mathrm{m}^{2}$ (level 5) in increments of $2.5 \mathrm{mg} / \mathrm{m}^{2}$ at each level. Three patients were assigned to each dose level. If no DLT was observed, the next level was opened. If the DLT was observed in one of three patients, three additional patients were accrued at this level. If no more DLT was observed, then the dose was escalated to the next level. If two or more patients at any dose level experienced the DLT, there was no further dose escalation.

If the patients experienced grade 4 esophagitis or other grade 4 radiation toxicities, the treatments including both

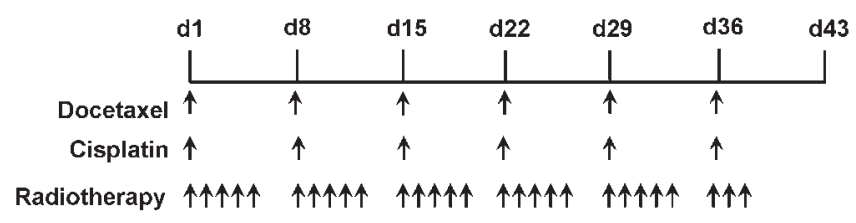

Figure 1. Treatment schedule of docetaxel and cisplatin with concurrent radiotherapy.

radiotherapy and chemotherapy were suspended. Treatments could recommence once reactions had improved to grade 1. Chemotherapy was ceased if grade 3 or 4 hematological toxicity, other grade 4 non-hematological toxicity occurred while radiotherapy could continue despite of chemotherapy modifications.

DLTs and MTD definition. Toxicities were assessed according to the National Cancer Institute Common Toxicity Criteria (NCI-CTC 3.0). Dose-limiting toxicity (DLT) were defined prospectively as at least one of the followings: (1) neutrophil count less than $0.5 \times 10^{9} / \mathrm{L}$ or neutrophil less than $1 \times 10^{9} / \mathrm{L}$ with fever; (2) platelet count less than $25 \times 10^{9} / \mathrm{L}$; (3) grade 4 radiation stomatitis; (4) any non-stomatitis grade $3 \sim 4$ radiation toxicity; (5) $\geq$ grade 3 nonhematologic toxicity. The maximum tolerated dose (MTD) was defined as the dose level that induced DLT in more than one-third of patients in a given cohort. The recommend dose of docetaxel for the phase II study was determined to that below the level of MTD.

Monitoring procedures and response assessment. Baseline evaluations were performed one week before study entry which included the complete history and physical examination, the PS assessment, the complete blood count, and the liver and renal function studies. Radiological assessment included the upper gastrointestinal barium X-ray, and the computed tomography (CT) scan of the chest and abdomen. Patients were required to undergo endoscopy with biopsy of the primary tumor within 4 weeks before study entry.

During treatments, patients were reviewed weekly, their weight was measured, PS assessed, physical examination and acute toxicities recorded. Complete blood count and biochemistry measurements were performed weekly. After the treatment was completed, patients were assessed clinically (physical examination, body weight and PS) at week 1, 2, and 4. Complete blood count and biochemistry were measured at week 2 and 4 . Patients underwent a chest/abdomen CT scan at week 4 . Follow-up evaluations were performed every 3 months until patient death or loss to follow-up, which included clinical examination, assessment of late radiation toxicity and repeated CT scan and endoscopy. RECIST Version 1.0 was used for assessment of radiographic response.

\section{Results}

Patient characteristics. A total of 21 patients were enrolled from three centers between October 2009 and May 2011. The 
clinical characteristics of the patients are summarized in Table 1. There are 4 female and 17 male, with median age of 61 years (from 46 to 70 years). The patient PS score was evaluated as 0 and 1 in 5 and 16 cases, respectively. There were 10 cases with severe tumor invasion to adjacent organ (T4), 6 cases with extensive local nodal disease (bulky N) and 5 cases with both.

Table 1. Patients characteristics

\begin{tabular}{lcc}
\hline Characteristic & Patients (N =21) & $\%$ \\
\hline Age (years) & & \\
$\quad$ Median & 61 & \\
$\quad$ Range & $46-70$ & \\
Sex & & \\
$\quad$ Male & 17 & 80.9 \\
$\quad$ Female & 4 & 19.1 \\
Performance status & & \\
$\quad$ (n) & 5 & 23.8 \\
$\quad$ & 16 & 76.2 \\
Histology & & \\
$\quad$ Well differentiated & 4 & 19.1 \\
$\quad$ Middle differentiated & 5 & 23.8 \\
$\quad$ Poor differentiated & 12 & 57.1 \\
Unresectable factors & & \\
$\quad$ Severe adjacent invasion (T4) & 10 & 47.6 \\
$\quad$ Bulky nodal metastasis (bulky N) & 6 & 28.6 \\
$\quad$ Both T4 and bulky N & 5 & 23.8 \\
Local symptoms & & \\
$\quad$ Incomplete obstruction & 2 & 9.5 \\
$\quad$ Pain & 15 & 71.4 \\
$\quad$ Bleeding & 3 & 14.3 \\
\hline
\end{tabular}

DLT and recommended dose. At least three patients were treated at each dose group. At the first $\left(5 \mathrm{mg} / \mathrm{m}^{2}\right)$, second $\left(7.5 \mathrm{mg} / \mathrm{m}^{2}\right)$ and third $\left(10 \mathrm{mg} / \mathrm{m}^{2}\right)$ docetaxel dose level, no patients developed DLT. At the fourth level (docetaxel $12.5 \mathrm{mg} / \mathrm{m}^{2}$ ), three patients were treated and one of them developed grade 3 neutropenia which required one week treatment delay. The cohort was then expanded to treat additional three patients. None of them developed DLT during treatments. The dose was then escalated to $15 \mathrm{mg} / \mathrm{m}^{2}$. Three patients were treated and one of them developed grade 3 neutropenia. When additional three patients were treated at the same level, two of them presented with DLTs with one grade- 4 neutropenia and one grade 3 nausea/vomiting. Therefore, level $4\left(15 \mathrm{mg} / \mathrm{m}^{2}\right)$ was regarded as the MTD and the recommended dose of docetaxel for the further phase II trial was determined to $12.5 \mathrm{mg} / \mathrm{m}^{2}$ weekly. Dose levels, patient enrolled and main specific hematological (neutropenia and anemia) and non-hematological (nausea/vomiting and anorexia) toxicities at each level were shown in Table 2 .

Overall toxicity. All patients were assessable for toxicity. The hematological and nonhematological toxicities in all patients encountered are shown in Table 3. Neutropenia and anemia were the most common hematological toxicities. $90.1 \%$ percent of patients $(n=19)$ experienced neutropenia. Of these, 9 patients (42.9\%) had grade 1, $6(28.6 \%)$ had grade 2 , $3(14.3 \%)$ had grade 3 , and 1 (4.8\%) had grade 4 neutropenia. The incidence of anemia was $95.2 \%$ with most grade $1-2$.

Nausea/vomiting, anorexia, mucositis and fatigue were the most common nonhematological toxicities. The incidence of nausea/vomiting was $85.7 \%$, with $11(52.4 \%)$ grade 1,6

Table 2. Dose escalation level, number of patients enrolled, and toxicity at each level

\begin{tabular}{|c|c|c|c|c|c|c|c|c|c|}
\hline \multirow{2}{*}{ Level } & \multirow{2}{*}{ Docetaxel $\left(\mathrm{mg} / \mathrm{m}^{2}\right)$} & \multirow{2}{*}{ Patients(n) } & \multicolumn{7}{|c|}{ Toxicity grade (n) } \\
\hline & & & & 0 & 1 & 2 & 3 & 4 & 5 \\
\hline \multirow[t]{4}{*}{1} & 5 & 3 & Neuropenia & 1 & 2 & 0 & 0 & 0 & 0 \\
\hline & & & Anemia & 1 & 2 & 0 & 0 & 0 & 0 \\
\hline & & & Nausea/vomiting & 1 & 2 & 0 & 0 & 0 & 0 \\
\hline & & & Anorexia & 1 & 2 & 0 & 0 & 0 & 0 \\
\hline \multirow[t]{4}{*}{2} & 7.5 & 3 & Neuropenia & 1 & 2 & 0 & 0 & 0 & 0 \\
\hline & & & Anemia & 0 & 2 & 1 & 0 & 0 & 0 \\
\hline & & & Nausea/vomiting & 1 & 2 & 0 & 0 & 0 & 0 \\
\hline & & & Anorexia & 1 & 1 & 1 & 0 & 0 & 0 \\
\hline \multirow[t]{4}{*}{3} & 10 & 3 & Neuropenia & 0 & 2 & 1 & 0 & 0 & 0 \\
\hline & & & Anemia & 0 & 1 & 2 & 0 & 0 & 0 \\
\hline & & & Nausea/vomiting & 1 & 1 & 1 & 0 & 0 & 0 \\
\hline & & & Anorexia & 1 & 1 & 1 & 0 & 0 & 0 \\
\hline \multirow[t]{4}{*}{4} & 12.5 & 6 & Neuropenia & 0 & 3 & 2 & 1 & 0 & 0 \\
\hline & & & Anemia & 0 & 4 & 2 & 0 & 0 & 0 \\
\hline & & & Nausea/vomiting & 0 & 4 & 2 & 0 & 0 & 0 \\
\hline & & & Anorexia & 0 & 3 & 3 & 0 & 0 & 0 \\
\hline \multirow[t]{4}{*}{5} & 15 & 6 & Neuropenia & 0 & 0 & 3 & 2 & 1 & 0 \\
\hline & & & Anemia & 0 & 4 & 2 & 0 & 0 & 0 \\
\hline & & & Nausea/vomiting & 0 & 2 & 3 & 1 & 0 & 0 \\
\hline & & & Anorexia & 0 & 4 & 2 & 0 & 0 & 0 \\
\hline
\end{tabular}



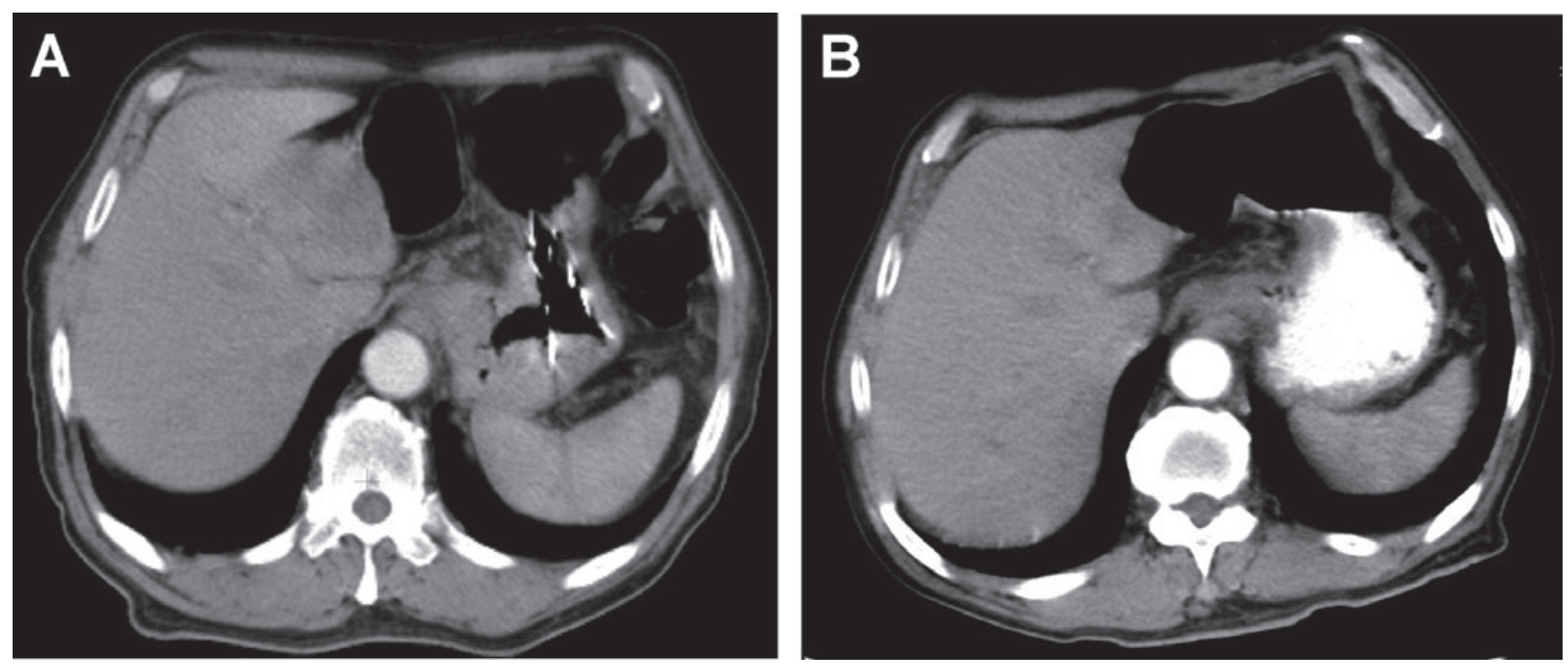

Figure 2. The CT images before (A) and after (B) treatments from representative case. The patient was a 58 years old male with locally advanced gastric adenocarcinoma. He received the treatments successfully according to protocol and was assessed as complete response with CT and endoscopic examination.

(28.6\%) grade 2 , and $1(4.8 \%)$ grade $3.85 .7 \%$ of patients experienced grade $1-2$ anorexia toxicities and received supportive parenteral nutrition. There were grade 1-2 mucositis (76.2\%), which was promptly resolved after appropriate support care, included omeprazole. Fatigue was also frequently found (76.2\%), commonly at grade 1-2.

Treatment Response. Although assessment of tumor response was not a primary objective of this study, it was performed in each patient. As patients were evaluable for radiologic response within the radiation field at 3 months, six patients achieved complete response (CR), eight patients had partial response (PR). The within-field response rate was then $66.7 \%$ (28.6\% CR and 38.1\% PR). The response data at different level was listed in Table 4. The CT images before and after treatments from representative case were shown in Figure 2.

\section{Discussion}

The management of locally advanced gastric cancer remains a challenge for both local-regional and systemic control. 5-FU and/or DDP-based combinations regimen are still the mainstay of the chemotherapy, used alone or combined with radiotherapy $[12,13]$. On the basis of the encouraging results observed as monotherapy, combinations of docetaxel with 5-FU and/or DDP have been investigated for gastric adenocarcinoma [13]. In present study, for the first time, we demonstrated that the concurrent administration of docetaxel, cisplatin and radiotherapy in patients with unresectable gastric adenocarcinoma is also feasible and tolerated. Although there were hematological and nonhematological toxicities, no treatment related mortality occurred. Most of nonhematological and hematological adverse effects were mild in our study. Alopecia, neuropathy, fluid retention, and nail change, which are frequently observed in high-dose docetaxel, did not occur at the treatment doses. Four of six patients experienced DLT at the docetaxel $15 \mathrm{mg} / \mathrm{m}^{2}$ weekly

Table 3. Overall Hematological and non-hematological toxicity

\begin{tabular}{lrrrrr}
\hline \multirow{2}{*}{ Toxicity } & \multicolumn{5}{c}{ Grade } \\
\cline { 2 - 6 } & 0 & 1 & 2 & 3 & 4 \\
\hline Leukocytopenia & 2 & 11 & 5 & 3 & 0 \\
Neutropenia & 2 & 9 & 6 & 3 & 1 \\
Lymphocytopenia & 4 & 10 & 7 & 0 & 0 \\
Thrombocytopenia & 7 & 11 & 3 & 0 & 0 \\
Anemia & 1 & 13 & 7 & 0 & 0 \\
Nausea/vomiting & 3 & 11 & 6 & 1 & 0 \\
Diarrhea & 15 & 3 & 3 & 0 & 0 \\
Anorexia & 3 & 12 & 6 & 0 & 0 \\
Mucositis & 5 & 9 & 7 & 0 & 0 \\
Fatigue & 5 & 11 & 5 & 0 & 0 \\
Dysphagia & 8 & 10 & 3 & 0 & 0 \\
Dehydration & 10 & 9 & 2 & 0 & 0 \\
\hline
\end{tabular}

Table 4. Treatment response at each dose level

\begin{tabular}{cccccc}
\hline \multirow{2}{*}{$\begin{array}{c}\text { Docetaxel } \\
\text { Dose level }\end{array}$} & Patients(n) & \multicolumn{4}{c}{ Treatment response } \\
\cline { 3 - 6 } & & CR & PR & SD & PD \\
\hline 1 & 3 & 0 & 2 & 0 & 1 \\
2 & 3 & 1 & 1 & 1 & 0 \\
3 & 3 & 1 & 0 & 1 & 1 \\
4 & 6 & 2 & 2 & 1 & 1 \\
5 & 6 & 2 & 3 & 1 & 0 \\
\hline
\end{tabular}


level, so $12.5 \mathrm{mg} / \mathrm{m}^{2}$ weekly was recommended for the further phase II/III study.

In order to improve the response and outcome of advanced gastric carcinoma, triplet chemotherapy regimens were also explored, including DCF (docetaxel, cisplatin and 5-Fu) and PCF (paclitaxel, cisplatin and 5-Fu) regimens. In a phase II trial comparing DCF, ECF (epirubicin, cisplatin and fluorouracil), and DC (docetaxel plus cisplatin) regimens for unresectable and/or metastatic gastric carcinoma, it was shown that DCF was superior to ECF in RR (36.6 vs. 25.0\%) and OS (10.4 vs 8.3 months) with acceptable toxicities [14]. In the phase III study (V325), it was reported that DCF regimen was effective in metastatic gastric cancer, with the response rates (RR) of $37 \%$, a median time to progression of 5.6 months, and a median survival of 9.2 months [15]. However, the high frequent grade 3-4 neutropenia (82\%) and diarrhea (19\%) made it intolerable when used in combination with radiotherapy. In a phase II trail [16] comparing PCF with PC (paclitaxel plus cisplatin), grade 3 or higher GI toxicity in the PCF group was 59\% at the interim analysis, which was significantly worse than that investigated at the other study [17]. The PCF arm was then closed. At the same time, $\mathrm{PC}$ regimen combined with radiation has been shown feasible and effective in palliative treatment for unresectable primary advanced or locally recurrent gastric adenocarcinoma [18]. Based on these findings, doublet but not the triplet regimen, docetaxel and cisplatin, was used in present study. Although concurrent chemoradiotherapy with DC regimen has not been studied in phase II/III trails for gastric cancer, other phase I trail using paclitaxel and cisplatin to combine with radiotherapy have shown encouraging results for gastric and esophageal cancer $[10,18]$.

When DC regimens are combined with radiotherapy, the toxicity related to two modalities is still concerned. To improve the tolerability of concurrent chemoradiotherapy, in present study the dose was modified. The DDP was fixed to lower dose compared with study from Ajani et al [19]. Docetaxel was still given weekly as previous studies $[10,14]$. All patients received effective supportive care, especially nutritional support, which was very important to maintain the tolerability of the patients for the combined treatments. In the setting of definitive chemoradiotherapy, image guided 3D-CRT and IMRT are warranted to appropriately protect the critical organs around the tumor, including small intestines, kidneys, liver and the spinal cord [20]. Typically the target volumes include gross tumor, nodal regions and structures at risk of involvement. In this study the GTV included only the primary tumor and involved lymph nodes, and the region of draining lymph nodes was not included in CTV. With this technique, the total dose is slightly improved to $50.4 \mathrm{~Gy}$ which is employed in other reports $[10,21]$. This dose is slightly higher as compared with 40 to 45 Gy in other Phase II-III studies in adjuvant or definitive settings [22,23]. Its goal is to improve the local controlling as well as optimize systemic therapy without increase of dose-limiting radiotherapy toxicities.

Concurrent chemoradiotherapy is recommended for patients with unresectable gastric cancer, which has shown to be advantageous over radiotherapy alone [4]. Radiotherapy is effective in controlling symptoms such as obstruction or hematemesis for gastric cancer. Cisplatin is a promising partner of docetaxel because of its established role as a radiosensitiser and minimal additional myelotoxicity. In addition to cytotoxic activity, docetaxel acts as an excellent radiation sensitizer, arresting cell-cycles in G2/M-phase, the most radiation-sensitive phase of the cell-cycle [24]. At the same time, the resistant to cisplatin and docetaxel is also concerned. Decreased intracellular concentration due to decreased drug uptake, increased reflux or increased inactivation by sulfhydryl molecules such as glutathione can cause resistance to cisplatin [25]. Mitochondrial DNA plays an important role in developing docetaxel resistance through the reduction of reactive oxygen species generation by regulating Fo-ATPase [26]. Further understanding of the drug resistance mechanism and its correlation with radiosensitivity may improve the effect of chemoradiotherapy for gastric cancer.

In recent years, progresses have been made in understanding the biological pathway and developing the molecular targeted therapy for gastric cancer. For example, trastuzumab in combination with chemotherapy is becoming the standard approach for the therapy of advanced HER2/neuoverexpressing upper gastrointestinal adenocarcinomas [27]. Immunohischemistry of HER-2 is not performed routinely in this study, but we will incorporate this in phase II study. The role of targeted therapy combined with chemoradiotherapy for gastric carcinoma need to be explored in further study.

In conclusion, modified DC regimen as weekly docetaxel and cisplatin combined with concurrent radiotherapy is feasible and safe for locally advanced gastric cancer. The overall response rates were $66.7 \%$. The results are encouraging. A phase II clinical trial is now underway in term of patient outcome.

\section{References}

[1] KAMANGAR F, DORES GM, ANDERSON WF. Patterns of cancer incidence, mortality, and prevalence across five continents: defining priorities to reduce cancer disparities in different geographic regions of the world. J Clin Oncol 2006; 24: 2137-2150. http: //dx.doi.org/10.1200/JCO.2005.05.2308

[2] SASAKO M. Principles of surgical treatment for curable gastric cancer. J Clin Oncol 2003; 21: 274s-275s. http: //dx.doi. org/10.1200/JCO.2003.09.172

[3] SWAN R, MINER TJ. Current role of surgical therapy in gastric cancer. World J Gastroenterol 2006; 12: 372-379.

[4] HENNING GT, SCHILD SE, STAFFORD, SL, DONOHUE JH, BURCH PA, et al. Results of irradiation or chemoirradiation for primary unresectable, locally recurrent, or grossly incomplete resection of gastric adenocarcinoma. Int J Radiat Oncol Biol Phys 2000; 46: 109-118. http: //dx.doi.org/10.1016/ S0360-3016(99)00379-X

[5] WAGNER AD, GROTHE W, HAERTING J, KLEBER G, GROTHEY A, et al. Chemotherapy in advanced gastric cancer: 
a systematic review and meta-analysis based on aggregate data. J Clin Oncol 2006; 24: 2903-2909. http: //dx.doi.org/10.1200/ LCO.2005.05.0245

[6] CHUN JH, KIM HK, LEE JS, CHOI JY, HWANGBO B, et al. Weekly docetaxel in combination with capecitabine in patients with metastatic gastric cancer. Am J Clin Oncol 2005; 28: 188194. http: //dx.doi.org/10.1097/01.coc.0000143877.53314.9c

[7] YOSHIDA K, NINOMIYA M, TAKAKURA N, HIRABAYASHI N, TAKIYAMA W, et al. Phase II study of docetaxel and S-1 combination therapy for advanced or recurrent gastric cancer. Clin Cancer Res 2006; 12: 3402-3407. http: //dx.doi. org/10.1158/1078-0432.CCR-05-2425

[8] THUSS-PATIENCE PC, KRETZSCHMAR A, REPP M, KINGREEN D, HENNESSER D, et al. Docetaxel and continuous-infusion fluorouracil versus epirubicin, cisplatin, and fluorouracil for advanced gastric adenocarcinoma: a randomized phase II study. J Clin Oncol 2005; 23: 494-501.

[9] ROTH AD, MAIBACH R, MARTINELLI G, FAZIO N, AAPRO MS, et al. Docetaxel (Taxotere)-cisplatin (TC): an effective drug combination in gastric carcinoma. Swiss Group for Clinical Cancer Research (SAKK), and the European Institute of Oncology (EIO). Ann Oncol 2000; 11: 301-306. http: //dx.doi.org/10.1023/A: 1008342013224

[10] DAY FL, LEONG T, NGAN S, THOMAS R, JEFFORD M, et al. Phase I trial of docetaxel, cisplatin and concurrent radical radiotherapy in locally advanced oesophageal cancer. Br J Cancer 2011; 104: 265-271. http: //dx.doi.org/10.1038/ sj.bjc. 6606051

[11] HIHARA J, YOSHIDA K, HAMAI Y, EMI M, YAMAGUCHI Y, et al. Phase I study of docetaxel (TXT) and 5-fluorouracil (5FU) with concurrent radiotherapy in patients with advanced esophageal cancer. Anticancer Res 2007; 27: 2597-2603.

[12] VAN CUTSEM E, VAN DE VELDE C, ROTH A, LORDICK F, $\mathrm{KOHNE} \mathrm{CH}$, et al. Expert opinion on management of gastric and gastro-oesophageal junction adenocarcinoma on behalf of the European Organisation for Research and Treatment of Cancer (EORTC)-gastrointestinal cancer group. Eur J Cancer 2008; 44: 182-194. http: //dx.doi.org/10.1016/j.ejca.2007.11.001

[13] MORABITO A, CARILLIO G, LONGO R. Systemic treatment of gastric cancer. Crit Rev Oncol Hematol 2009; 70: 216-234. http: //dx.doi.org/10.1016/j.critrevonc.2008.08.005

[14] ROTH AD, FAZIO N, STUPP R, FALK S, BERNHARD J, et al. Docetaxel, cisplatin, and fluorouracil; docetaxel and cisplatin; and epirubicin, cisplatin, and fluorouracil as systemic treatment for advanced gastric carcinoma: A randomized phase II trial of the Swiss Group for Clinical Cancer Research. J Clin Oncol 2007; 25: 3217-3223. http: //dx.doi.org/10.1200/ LCO.2006.08.0135

[15] VAN CUTSEM E, MOISEYENKO VM, TJULANDIN S, MAJLIS A, CONSTENLA M, et al. Phase III study of docetaxel and cisplatin plus fluorouracil compared with cisplatin and fluorouracil as first-line therapy for advanced gastric cancer: a report of the V325 Study Group. J Clin Oncol 2006; 24: 4991-4997. http: //dx.doi.org/10.1200/JCO.2006.06.8429

[16] SCHWARTZ GK, WINTER K, MINSKY BD, CRANE C, THOMSON PJ, et al. Randomized phase II trial evaluating two paclitaxel and cisplatin-containing chemoradiation regimens as adjuvant therapy in resected gastric cancer (RTOG-0114). J Clin Oncol 2009; 27: 1956-1962. http: //dx.doi.org/10.1200/ LCO.2008.20.3745

[17] MACDONALD JS, SMALLEY SR, BENEDETTI J, HUNDAHL SA, ESTES NC, et al. Chemoradiotherapy after surgery compared with surgery alone for adenocarcinoma of the stomach or gastroesophageal junction. N Engl J Med 2001; 345: 725-730. http: //dx.doi.org/10.1056/NEJMoa010187

[18] YOSHIKAWA T, TSUBURAYA A, HIRABAYASHI N, YOSHIDA K, NAGATA N, et al. A phase I study of palliative chemoradiation therapy with paclitaxel and cisplatin for local symptoms due to an unresectable primary advanced or locally recurrent gastric adenocarcinoma. Cancer Chemother Pharmacol 2009; 64: 1071-1077. http: //dx.doi.org/10.1007/ s00280-009-0963-3

[19] AJANI JA, MANSFIELD PF, CRANE CH, WU TT, LUNAGOMEZS, et al. Paclitaxel-based chemoradiotherapy in localized gastric carcinoma: degree of pathologic response and not clinical parameters dictated patient outcome. J Clin Oncol 2005; 23: 1237-1244. http: //dx.doi.org/10.1200/JCO.2005.01.305

[20] MINN AY, HSU A, LA T, KUNZ P, FISHER GA, et al. Comparison of intensity-modulated radiotherapy and 3-dimensional conformal radiotherapy as adjuvant therapy for gastric cancer. Cancer 2011; 116: 3943-3952.

[21] SUN W, METZ JM, GALLAGHER M, O'DWYER PJ, GIANTONIO B, et al. Two phase I studies of concurrent radiation therapy with continuous-infusion 5-fluorouracil plus epirubicin, and either cisplatin or irinotecan for locally advanced upper gastrointestinal adenocarcinomas. Cancer Chemother Pharmacol 2011; 67: 621-627. http: //dx.doi.org/10.1007/ s00280-010-1365-2

[22] SAIKAWA Y, KUBOTA T, KUMAGAI K, NAKAMURA R, KUMAI K, et al. Phase II study of chemoradiotherapy with S-1 and low-dose cisplatin for inoperable advanced gastric cancer. Int J Radiat Oncol Biol Phys 2008; 71: 173-179. http: //dx.doi.org/10.1016/j.ijrobp.2007.09.010

[23] BAMIAS A, KARINA M, PAPAKOSTAS P, KOSTOPOULOS I, BOBOS M, et al. A randomized phase III study of adjuvant platinum/docetaxel chemotherapy with or without radiation therapy in patients with gastric cancer. Cancer Chemother Pharmacol 2010; 65: 1009-1021. http: //dx.doi.org/10.1007/ $\underline{\mathrm{s} 00280-010-1256-6}$

[24] KIM ES, KHURI FR. Docetaxel and radiation as combinedmodality therapy. Oncology (Williston Park) 2002; 16: 97-105.

[25] KARTALOU M, ESSIGMANN JM. Mechanisms of resistance to cisplatin. Mutation Research 2001; 478: 23-43 http: //dx.doi. org/10.1016/S0027-5107(01)00141-5

[26] MIZUMACHIT, SUZUKIS, NAITO A, CARCEL-TRULLOLS J, EVANS TT, et al. Increased mitochondrial DNA induces acquired docetaxel resistance in head and neck cancer cells. Oncogene. 2008; 27: 831-838 http: //dx.doi.org/10.1038/ sj.onc. 1210681

[27] KAUR A, DASANU CA. Targeting the HER2 pathway for the therapy of lower esophageal and gastric adenocarcinoma. Expert Opin Pharmacother. 2011; 12: 2493-2503 http: //dx.doi. org/10.1517/14656566.2011.605354 